\title{
Simulation-Based Optimal Portfolio Selection Strategy—Evidence from Asian Markets
}

\author{
Longqing Li \\ Correspondence: Longqing Li, Department of Economics, Christopher Newport University, Newport News, VA, USA.
}

Received: June 19, 2018

doi:10.11114/aef.v5i5.3376
Accepted: July 10, $2018 \quad$ Available online: July 13, 2018

URL: https://doi.org/10.11114/aef.v5i5.3376

\begin{abstract}
Recently portfolio optimization has become widely popular in risk management, and the common practice is to use mean-variance or Value-at-Risk (VaR), despite the VaR being incoherent risk measure because of the lack of subadditivity. This has led to the emergence of the conditional value-at-risk (CVaR) approach, consequently, a gradual development of mean-CVaR portfolio optimization. To seek an optimal portfolio selection strategy and increase the robustness of the result, the paper studies the performance of portfolio optimization in Asian markets using a Monte-Carlo simulation tool, creates a variety of randomly selected portfolios that consists of Asian ADRs listed in NYSE from 2011 to 2016, and applies both optimization frameworks with different skewed fat-tailed distributions, including the Generalized Hyperbolic (GH) and skewed-T distribution. The main result shows that the Generalized Hyperbolic distribution produces the lowest risk under a given rate of return, while the skewed-T distribution creates a diversification allocation outcome similar to that of historical simulation.
\end{abstract}

Keywords: portfolio optimization, mean-CVaR, Monte Carlo, hyperbolic distribution

\section{Introduction}

In recent years, portfolio optimization has become one of the most important areas in financial risk management. Often, a financial analyst wants to minimize the risk for a given rate of return, or maximize the return given a desired risk level. Empirically, the most commonly used tool is a mean-variance, developed by Markowitz (1952), which applies quadratic programming to minimize the variance of the portfolio. In other words, it uses the variance to measure the portfolio risk. Nevertheless, the drawback of this approach, as explained by Markowitz, is the fact that variance does not differentiate a positive shock from a negative one. It treats the downside and upside risk in the same way. Moreover, another limitation of the mean-variance approach is that the model only works for a multivariate normal distribution. Such limitations weaken the applicability of mean-variance analysis, because it is well understood that the returns of financial markets are often not normally distributed, but exhibit excess kurtosis and skewness.

To overcome the issues inherent in mean-variance approach, a separate measure, called Value-at-Risk (VaR), has become increasingly popular. The VaR is essentially the quantile of the loss function, measured as the worst loss to be expected over a specified time period under a certain confidence level. It enables the investor and portfolio analyst to better understand the scope of the worst loss under a different probability when triggered by downside risk. The weakness of $\mathrm{VaR}$ is that it only provides the minimum of the worst loss, rather than the actual loss beyond that level.

More importantly, the VaR is not a coherent risk measure due to the lack of subadditivity. Acerbi and Tasche (2002) document that a risk measure, to be coherent, must satisfy four properties: monotonicity, positively homogeneity, translation invariance and sub-additivity. Sub-additivity means the risk of a portfolio made of two assets should be no more than the sum of their individual asset risks. In mathematical terms, it is $\rho(X+Y) \leq \rho(X)+\rho(Y)$ (where $\rho$ represents the risk measure). Put differently, sub-additivity is a fundamental and essential property in portfolio selection because it represents an investment principle: portfolio diversification contributes to risk reduction.

The incoherence of VaR has led many to use an alternative, the conditional value-at-risk (CVaR), aka expected shortfall. The popularity of CVaR lies in two important areas: First, it possesses all the properties of a coherent risk measure. Second, CVaR provides a more comprehensive look at the worst-case loss since it measures the average loss of the tail distribution under different scenarios. The $\mathrm{CVaR}$ is an extension of the VaR that equals to the expected value of loss given the exceedance, so it is the conditional tail expectation of VaR. Rockafellar and Uryasev (2000) compare the performance of mean-variance and mean-CVaR, and find the issue can be formulated as a linear programming problem. 
Alexander and Baptista (2004) analyze portfolio optimization in mean-variance model with a VaR or CVaR constraint and show that CVaR constraint is much effective to control slightly risk-free agents for a given confidence level. Ho, Cadle and Theobald (2008) evaluate the performance between CVaR, VaR and variance in portfolio optimization and demonstrate that CVaR is a better framework when dealing with a fat-tailed distribution.

The popularity of a coherent risk measure such as CVaR has induced extensive study of the mean-CVaR portfolio optimization. To have a robust portfolio selection strategy, and address the existence of high kurtosis and skewness, this paper suggests using a simulation model to find the optimal portfolio allocation when applied with various skewed fat-tailed distributions on both optimization frameworks.

This paper differs from other literatures about portfolio optimization in three important ways. First, it relaxes the constraint on return governed by a log-normal distribution. Second, it increases the robustness of the result using Monte Carlo simulation to random select a new portfolio, and analyzes the optimal allocation on both optimization frameworks, mean-variance and mean-CVaR. Third, it introduces a Generalized Hyperbolic (GH) and a skewed-T distribution to address the issues of non-normality, skewness and kurtosis.

The remainder of the paper is organized as follows. Section 2 describes the background of the portfolio selection, mean-variance and mean-CVaR models. Section 3 describes the dataset and presents stylized findings. Section 4 introduces the various skewed heavy-tailed distributions and shows the result of optimal allocation under different cases. Section 5 concludes.

\section{Portfolio Selection}

\subsection{Markowitz Mean-Variance Optimization Framework}

The basic idea of mean-variance analysis is to maximize the expected return of a portfolio for a given level of variance (risk). It can be represented by mathematical form as follows:

$$
\max E\left[w^{T} R\right]
$$

subject to $w^{T} \sum w<=T$ and $\sum_{i=1}^{n} w_{i}=V_{0}$

where $w$ is a vector of portfolio holdings of each asset, $\Sigma$ is the variance-covariance matrix of returns for each asset, $w^{T} R$ is the targeted return of the portfolio, and $\mathrm{R}$ is a vector of expected return.

An alternative version of the model is mean-variance trade-off formulation, that is,

$$
\max w^{T} \mu-\frac{c}{2 V_{0}} w^{T} \sum w
$$

subject to $\sum_{i=1}^{n} w_{i}=V_{0}$

where $\mu$ is a vector of mean log-return of portfolio and $\mathrm{c}$ is a risk aversion coefficient.

A commonly used practice in portfolio allocation is a restriction on short positions. In other words, only the long position is allowed in investing $\left(\boldsymbol{W}_{\boldsymbol{i}}>0\right)$. As a result, a constrained mean-variance optimization becomes:

$$
\max w^{T} \mu-\frac{c}{2 V_{0}} w^{T} \sum w
$$

subject to $\sum_{i=1}^{n} w_{i}=V_{0}$ and $w_{i}>=0, i=1, \ldots, n$

\subsection{Mean-CVaR Optimization Framework}

The CVaR is the conditional tail distribution of $\mathrm{VaR}$ and can be expressed by

$$
\operatorname{CVaR}_{p}(X)=\frac{1}{p} \int_{\mathrm{o}}^{p} \operatorname{VaR}(X) d u=\frac{1}{1-q} \int_{q}^{1} V a R_{u}(L) d u
$$

where $q=1-p, L$ is the discounted loss. The VaR of a stochastic variable $X$ is defined as the (1-p) level quantile of associated discounted loss L. Hence CVaR is an extension of $\mathrm{VaR}$, and its sub-additivity property makes it to be a coherent risk measure than down-side risk VaR.

To minimize the CVaR, we impose the constraints that initial capital must be fully invested in the assets $\left(\sum_{i=1}^{n} w_{i}=V_{0}\right)$ and no short position is allowed in investment, therefore the mean-CVaR optimization can be written as

$$
\min C V a R_{p}\left(w^{T} R\right)
$$

subject to $w^{T} \mu>=\theta$

$\sum_{i=1}^{n} w_{i}=V_{0}$ and $w_{i}>=0, i=1, \ldots, n$ 
where $\boldsymbol{\theta}$ is the tolerance level of expected return of portfolio with $\mathrm{n}$ assets, $\mathrm{p}$ is p-level loss quantile.

The technical challenge in the equation above is that $\mathrm{CVaR}$ is an integral of $\mathrm{VaR}$, meaning optimizing CVaR requires VaR calculated first. Rockafellar and Uryasev (2000) contribute to solving this issue by introducing the independence of the CVaR and VaR functions, simplifying the optimization programming process. In other words, CVaR could be calculated without first having to know VaR on which its definition depends. A central piece is to characterize the $\mathrm{CVaR}_{\mathrm{p}}$ and $\mathrm{VaR}_{\mathrm{p}}$ with an auxiliary function $\mathrm{H}_{\mathrm{p}}$, defined by

$$
\begin{gathered}
H_{p}(w, \alpha)=\alpha+\frac{1}{1-q} \int[L(w, R)-\alpha)^{+} f(R) d R \\
\text { where }[x]^{+}= \begin{cases}x & x \geq 0 \\
0 & x \leq 0\end{cases}
\end{gathered}
$$

and $f(R)$ be the probability density function of the return vector R. With the help of the auxiliary variable, the mean-CVaR portfolio allocation can be stated as

$$
\begin{gathered}
\min _{w, \alpha} \alpha+\frac{1}{(1-q) D} \sum_{d=1}^{D} z_{d} \\
\text { subject to } w^{T} \mu \geq \theta, z_{d} \geq 0,-L\left(w, R_{d}\right)+\alpha+z_{d} \geq 0
\end{gathered}
$$

and $\sum_{i=1}^{n} w_{i}=V_{0}, w_{i} \geq 0$, where $Z_{d}$ is the auxiliary variable $(\mathrm{d}=1, \ldots, \mathrm{D})$, and $\mathrm{L}$ is the discounted loss. With convex function $\mathrm{L}(\mathrm{w}, \mathrm{R})$, the optimization problem becomes a convex linear program ${ }^{1}$.

\section{Dataset}

To show the effect of exchange-rate risk in portfolio allocation, the paper uses the daily stock prices of foreign ADR (non-US issuers) currently listed in NYSE from 2011/01/01 to 12/31/2016. In August 2011, global stock market went through a sharp drop in prices affecting China and Japan. Also, in 2011, a magnitude 9.0 earthquake and tsunami struck the pacific coast of Tohohu and causes a deep impact on Japanese stock market. Between 2015 and 2016, Chinese stock market experienced a significant turbulence, with a third of the value of A-shares on the Shanghai Stock Exchange was wiped out within a month. Some call the major aftershocks as the Black Monday. In 2016, Japan went through one of its worst stock market slumps led by Nikkei 225 index. Considering China and Japan to be the two largest economies behind U.S., with enormous influence on Asian financial markets, we are particularly interested in these two countries stocks with different market capitalization. To make the selection more representative, we pick stocks from different sectors and industries. As a result, we narrow the list to 11 stocks and calculate each asset's logarithm return to have a daily time series spanning six years. These stocks are CEA, SNP, HNP, PTR, BIDU, SINA, HMC, MTU, CAJ, TM and NTT. The first six stocks are from China, and the last 5 stocks are from Japan. It covers industries in travel and leisure, oil and gas, mobile telecommunication, electricity, automobile parts, technology hardware, banks and fixed line communication. Table 1 lists each ADR's symbol, company name and associated industry.

Table 1. Asset Symbol, Name of Company and Industry

\begin{tabular}{llll}
\hline Symbol & Company & Industry & Listed \\
\hline NTT & Nippon Telegraph and Telephone Corporation & Fixed Line Telecommunications & $9 / 29 / 94$ \\
CAJ & Canon Inc. & Technology Hardware \& Equipment & $9 / 14 / 00$ \\
TM & Toyota Motors Corporation & Automobile \& Parts & $9 / 29 / 99$ \\
HNP & Huaneng Power International Inc. & Electricity & $10 / 6 / 94$ \\
SNP & China Petroleum \& Chemical Corp. & Oil \& Gas Producers & $10 / 18 / 00$ \\
BIDU & Baidu Inc. & Internet Content \& Information & $8 / 1 / 05$ \\
HMC & Honda Motors Co., Ltd. & Automobile \& Parts & $2 / 11 / 77$ \\
CEA & China Eastern Airline Co., Ltd. & Travel and Leisure & $2 / 4 / 97$ \\
PTR & PetroChina Company Limited & Oil \& Gas Producers & $4 / 6 / 00$ \\
SINA & SINA Corporation & Internet Content \& Information & $4 / 3 / 00$ \\
MTU & Mitsubishi UFJ Financial Group & Banks & $9 / 19 / 89$ \\
\hline
\end{tabular}

${ }^{1}$ See Skoglund and Chen (2009) 
Figure 1 provides the historical stock price movement.

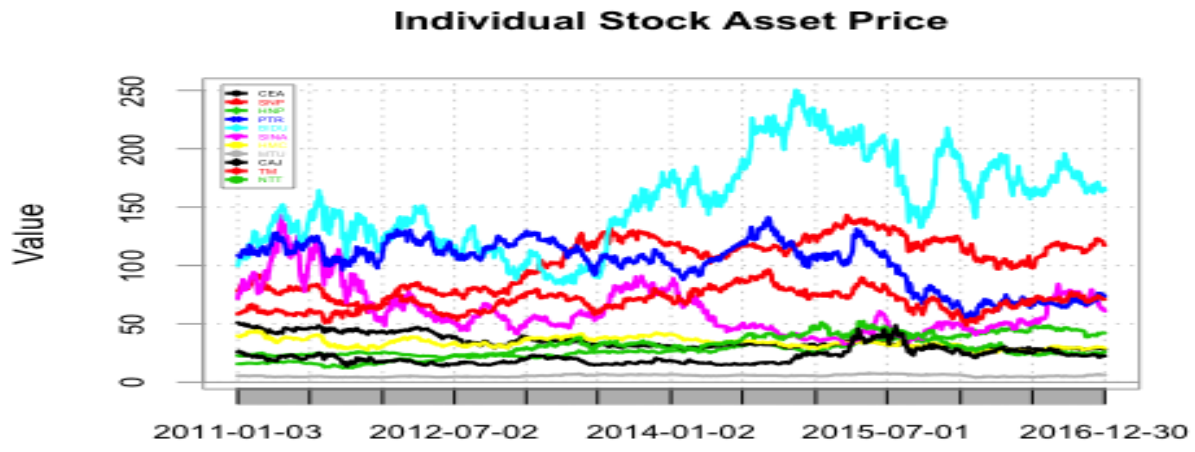

Figure 1. Individual Asset Price From 2011 To 2016

Figures 2.1 and 2.2 plot the daily asset returns in China and Japan, respectively.

Figure 2.1 Daily Returns of Asset in China(in \%)

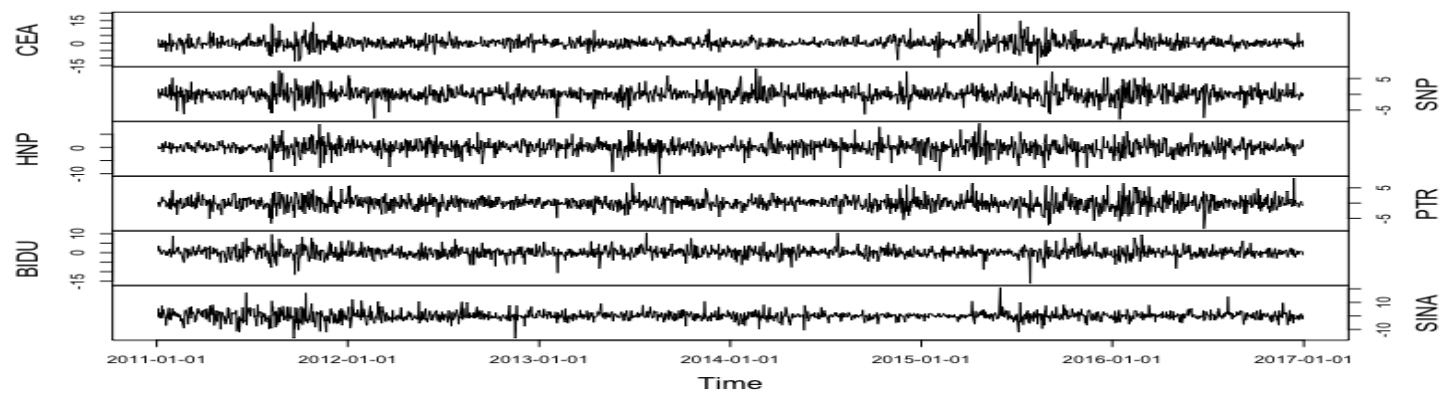

Figure 2.2 Daily Returns of Asset in Japan(in \%)

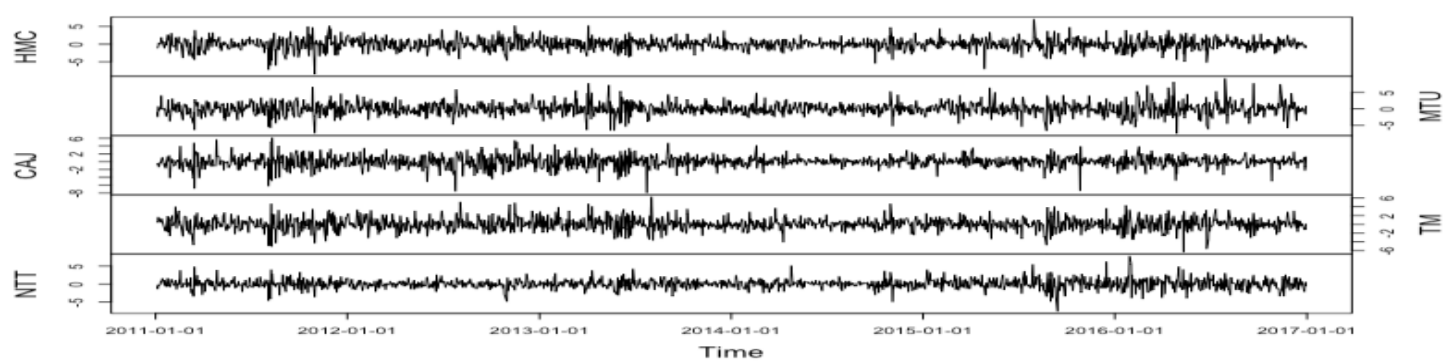

Table 2 provides the statistical summary of all assets' daily returns

Table 2. Summary Statistics of Individual Asset ${ }^{2}$

\begin{tabular}{|c|c|c|c|c|c|c|c|c|c|c|c|}
\hline & CEA & SNP & HNP & PTR & BIDU & SINA & HMC & MTU & CAJ & TM & NTT \\
\hline OBSERVATION & 1509 & 1509 & 1509 & 1509 & 1509 & 1509 & 1509 & 1509 & 1509 & 1509 & 1509 \\
\hline MINIMUM & -14.51 & -7.97 & -10.17 & -8.44 & -16.25 & -16.45 & -8.49 & -7.46 & -7.92 & -6.28 & -5.34 \\
\hline QUARTILE 1 & -1.63 & -1.04 & -1.13 & -1.12 & -1.33 & -1.52 & -0.90 & -1.09 & -0.83 & -0.76 & -0.74 \\
\hline MEDIAN & -0.18 & 0.01 & 0.04 & -0.03 & -0.11 & -0.06 & 0.00 & 0.00 & 0.00 & 0.00 & 0.04 \\
\hline $\begin{array}{l}\text { ARITHMETIC } \\
\text { MEAN }\end{array}$ & -0.01 & 0.01 & 0.03 & -0.03 & 0.03 & -0.01 & -0.02 & 0.01 & -0.04 & 0.03 & 0.04 \\
\hline QUARTILE 3 & 1.52 & 1.00 & 1.30 & 1.03 & 1.40 & 1.53 & 0.90 & 1.08 & 0.80 & 0.80 & 0.78 \\
\hline MAXIMUM & 19.27 & 8.09 & 9.09 & 8.15 & 10.44 & 20.92 & 7.04 & 9.24 & 6.10 & 6.21 & 5.92 \\
\hline SE MEAN & 0.08 & 0.05 & 0.06 & 0.05 & 0.06 & 0.08 & 0.04 & 0.05 & 0.04 & 0.04 & 0.03 \\
\hline VARIANCE & 8.85 & 3.34 & 4.75 & 3.34 & 5.98 & 10.43 & 2.39 & 3.51 & 2.13 & 1.90 & 1.70 \\
\hline STDEV & 2.98 & 1.83 & 2.18 & 1.83 & 2.44 & 3.23 & 1.55 & 1.87 & 1.46 & 1.38 & 1.31 \\
\hline SKEWNESS & 0.44 & 0.05 & -0.26 & 0.09 & -0.09 & 0.25 & -0.24 & 0.17 & -0.38 & 0.01 & 0.06 \\
\hline KURTOSIS & 3.50 & 2.00 & 1.92 & 1.44 & 3.13 & 4.13 & 2.00 & 2.16 & 2.89 & 1.49 & 1.41 \\
\hline JARQUE.BERA & 819.41 & 251.50 & 247.62 & 131.51 & 618.17 & 1089.84 & 266.77 & 300.83 & 563.41 & 140.33 & 125.53 \\
\hline
\end{tabular}

2 The observations cover from 01/01/20111 to 12/31/2016, all the numbers are measured in absolute units. 
It is shown that each asset's distribution is leptokurtic and asymmetric given the high excess kurtosis and skewness. In addition, a high Jarque-Bera test statistic suggests the non-normality of most of the distributions. The statistical results manifest the important properties of return distributions, thus motivate us to use a heavy-tailed skewed probability density function in the portfolio selection.

Figure 3. Describes the relationship between volatility and return in portfolio optimization.

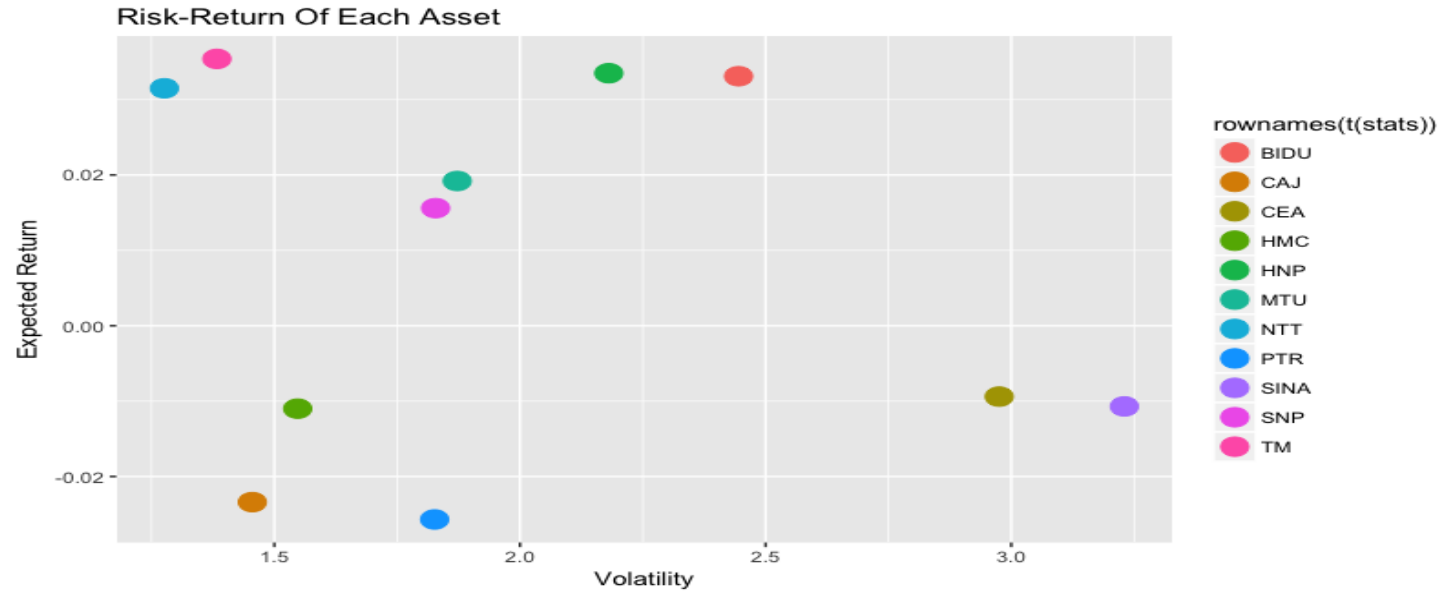

Figure 3. Risk-Expected Return of Each Asset ${ }^{3}$

An ideal asset is one that has lower volatility and higher returns, so is located on the top left side of the grid. As can be seen, NTT and TM are the most attractive assets because both are the more likely to yield a high amount of positive return per unit of volatility.

\section{Portfolio Allocation Result}

\subsection{Asset-Allocation with Historical Distribution}

We first use the historical stock returns as an input to determine the optimal asset allocation using mean-variance and mean-CVaR. To be consistent, we use the full-sample period under a 95\% confidence level with long only position because it is the standard level for internal risk management. To determine the weight of optimal allocation, we use the global minimum variance (GMV) obtained from the efficient portfolio frontier. In other words, the share of each asset in the optimal portfolio is selected from the portfolio with the lowest risk on the efficient frontier. Figure 4.1 displays the optimal allocation using the two frameworks.

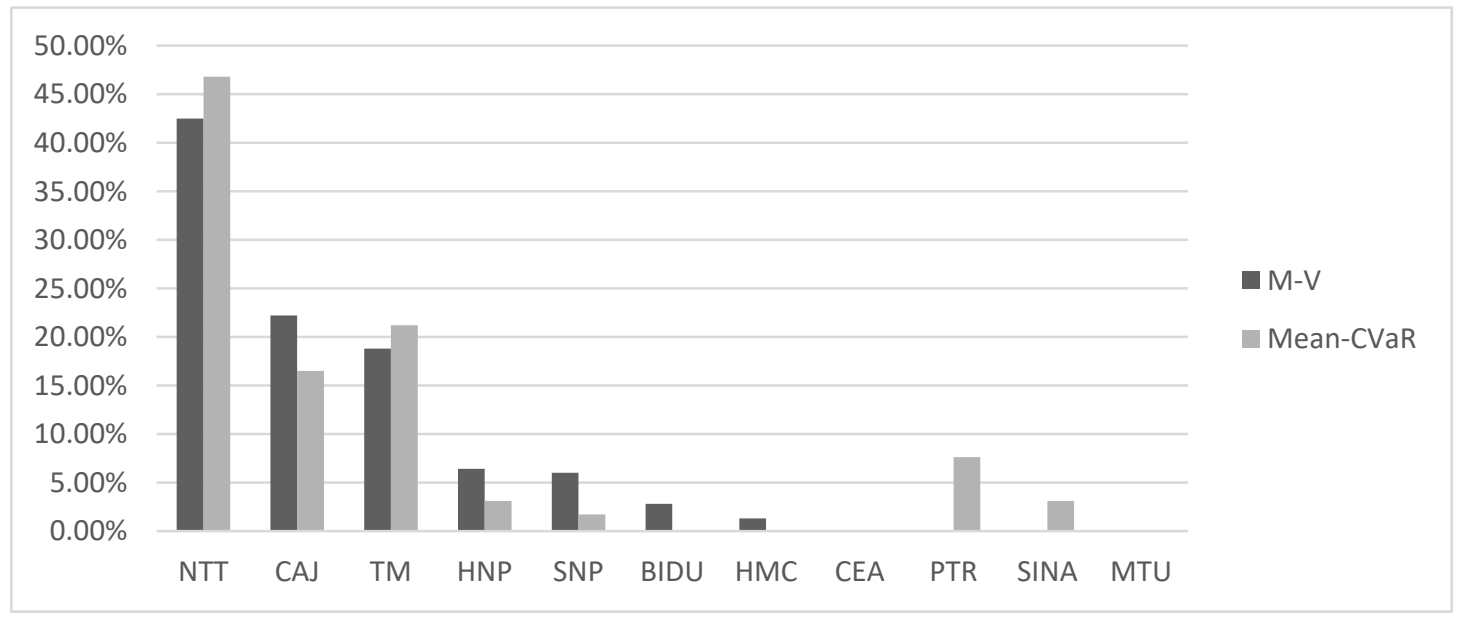

Figure 4.1. Asset Allocation using Historical Approach

A high percentage of NTT, TM and CAJ in the optimal allocation in both frameworks reflects the benefit of investing in high risk-premium stocks. NTT stock comes on top with a controlling share of $43 \%$ in mean-variance framework. In

3 The expected return and volatility are measured in absolute terms. 
addition, the historical distribution method shows that there is no strong evidence suggesting any difference between two frameworks in optimal allocation.

\subsection{Asset Allocation with Generalized Hyperbolic (GH) Distribution}

To address the heavy tails and skewness in the financial returns, some suggest using Variance Gamma distributed increments with a Levy process, while others opt for the hyperbolic distribution, or the Normal Inverse Gaussian distribution. All these belong to a subclass of Generalized Hyperbolic distribution (GH) introduced by Barndorff-Nielsen (1977). The Generalized Hyperbolic distribution is a part of the Multivariate Normal Mean-Variance Mixture (MNMVM) distribution that can be defined as follows:

$$
X=\mu+W \gamma+\sqrt{(} W) A Z
$$

where $\mu, \gamma \epsilon R^{d}$ and $Z-N_{k}\left(0, I_{k}\right)$ is a standard multivariate normal random variable and $\mathrm{W}$ is a non-negative mixing random variable, $\left\lfloor\in \in R^{a * k}\right.$ is a distribution parameter, $\Sigma=A * A^{\prime}$ requires to be positive definite.

A GH-distributed random variable is the one with above representation with $\mu$ as the location parameter, $\gamma$ as the skewness parameter, $\Sigma$ as the scale parameter and $W$ the shock factor for skewness and scale. Birge and Chavez-Bedoya (2016) show the optimal portfolio allocation could be found in both close-form and a successive approximation scheme when asset returns follow a generalized hyperbolic skewed $t$ distribution with exponential utility function.

Here we apply Monte Carlo to generate 1000 simulated price of each stock using the estimated four moments from GH distribution. First, we determine the estimated moments using historical stock returns. Second, we create a randomized portfolio based on the estimated four moments. Third, we repeat the previous step 1000 times to have 1000 simulated stock returns. Figure 4.2 displays the optimal asset allocation between the two frameworks when applying a heavy-tailed skewed GH distribution.

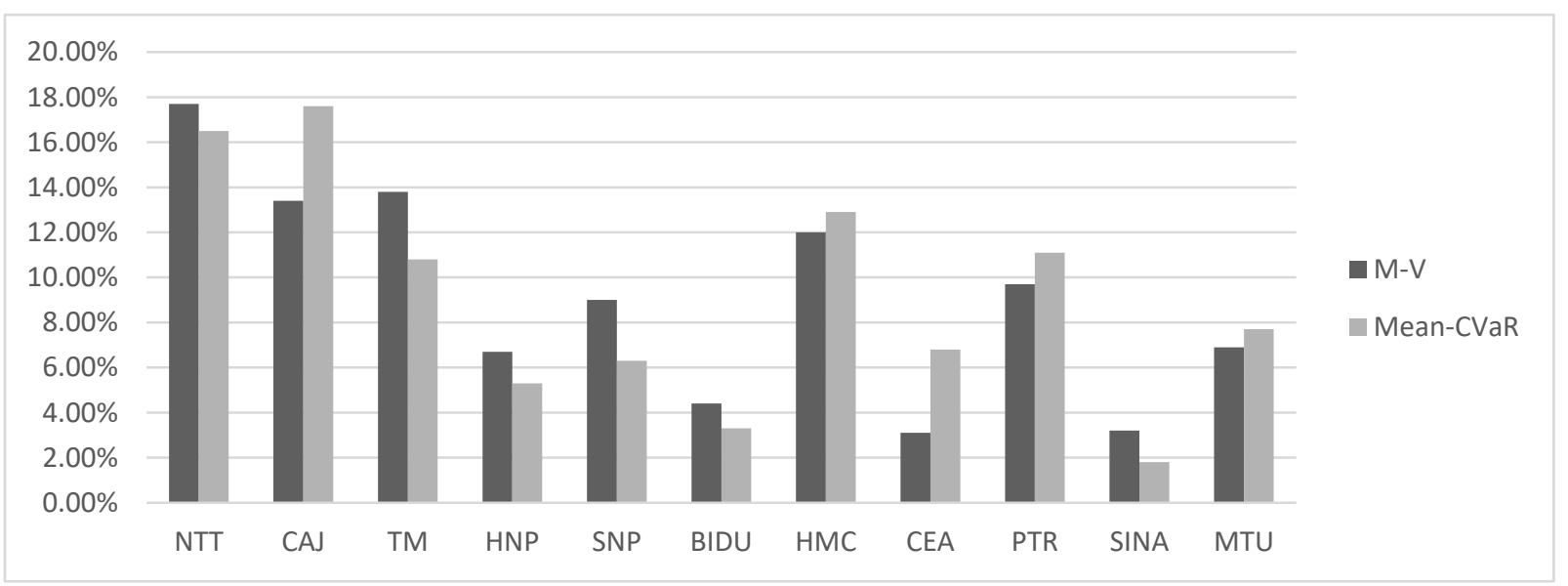

Figure 4.2. Asset Allocation using GH Distribution

In a stark contrast to the historical approach, the new optimal portfolio gives a high degree of diversification. The result is a more diversified portfolio with an approximately equal amount of shares of each asset. Despite NTT, TM and CAT remains the top three biggest stock holdings, the share of their combined contributions decreases to less than 50\%. A moderate increase in holding more stock highlights the fundamental principle, that is, the diversification lowers the portfolio risk, as defined here.

\subsection{Asset Allocation with Skewed-T Fat-Tailed Distribution}

\subsubsection{Scenario Analysis One}

In the first scenario, we explore the impact of a simulated return using a skewed-t distribution with optimal degrees of freedom, that is, the degrees of freedom are self-determined based on the Akaike information criterion (AIC). The degrees of freedom determine the thickness of the tail, and the t-distribution resembles normal distribution when it increases. Determining an appropriate shape parameter is an important step in doing the portfolio selection. In that regard, we use the optimal degrees of freedom that minimizes the information criterion AIC. We choose these distributions because they have the ability to capture the properties of the empirical distribution. Figure 4.3.1 compares the performance of asset allocation between two optimization frameworks. 


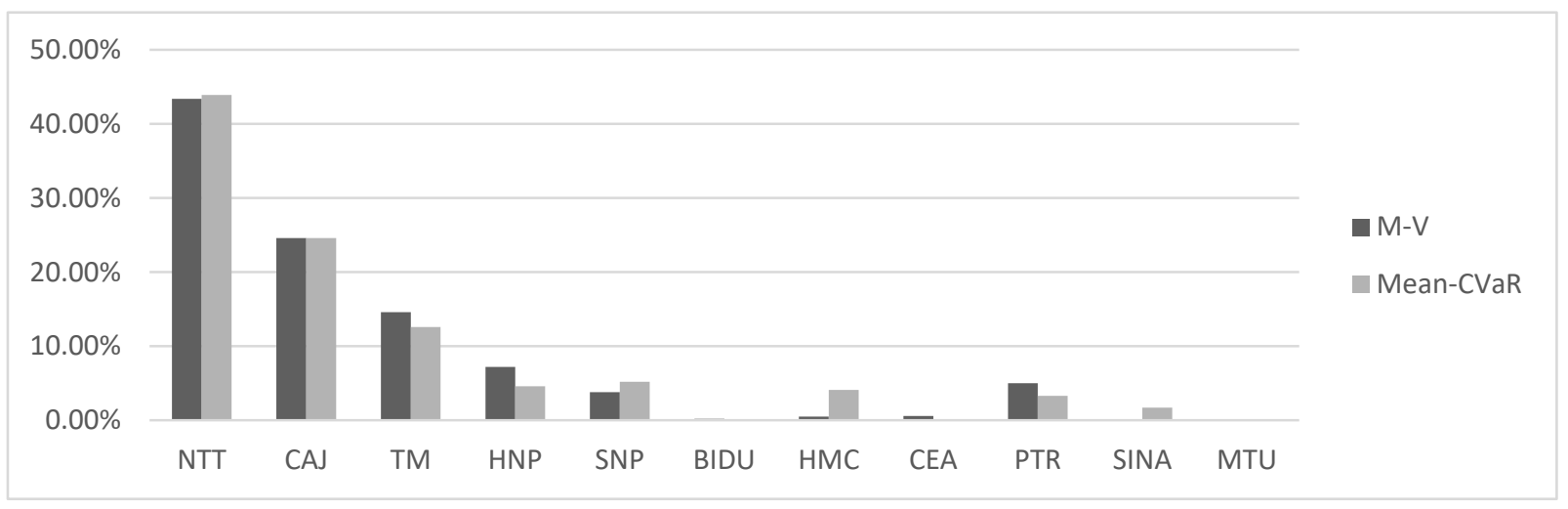

Figure 4.3.1 Asset Allocation from Optimal Skewed T

Mean-CVaR is shown to include more assets in the allocation outcome than the mean-variance framework, as evidenced by an inclusion of high volatility asset like SINA. For the most part, the optimal allocation resulting from the optimal skewed-t distribution resembles that of the historical distribution.

\subsubsection{Scenario Analysis Two}

To further explore the effect of thickness of tail on the portfolio selection, we perform a second scenario analysis that presumes the simulated returns generated from skewed-t distributions with 2 and 3 degrees of freedom, respectively. As discussed earlier, a small degree of freedom implies a fatter tail in the distribution.

Figure 4.3.2 shows the optimal asset allocation with 2 degrees of freedom.

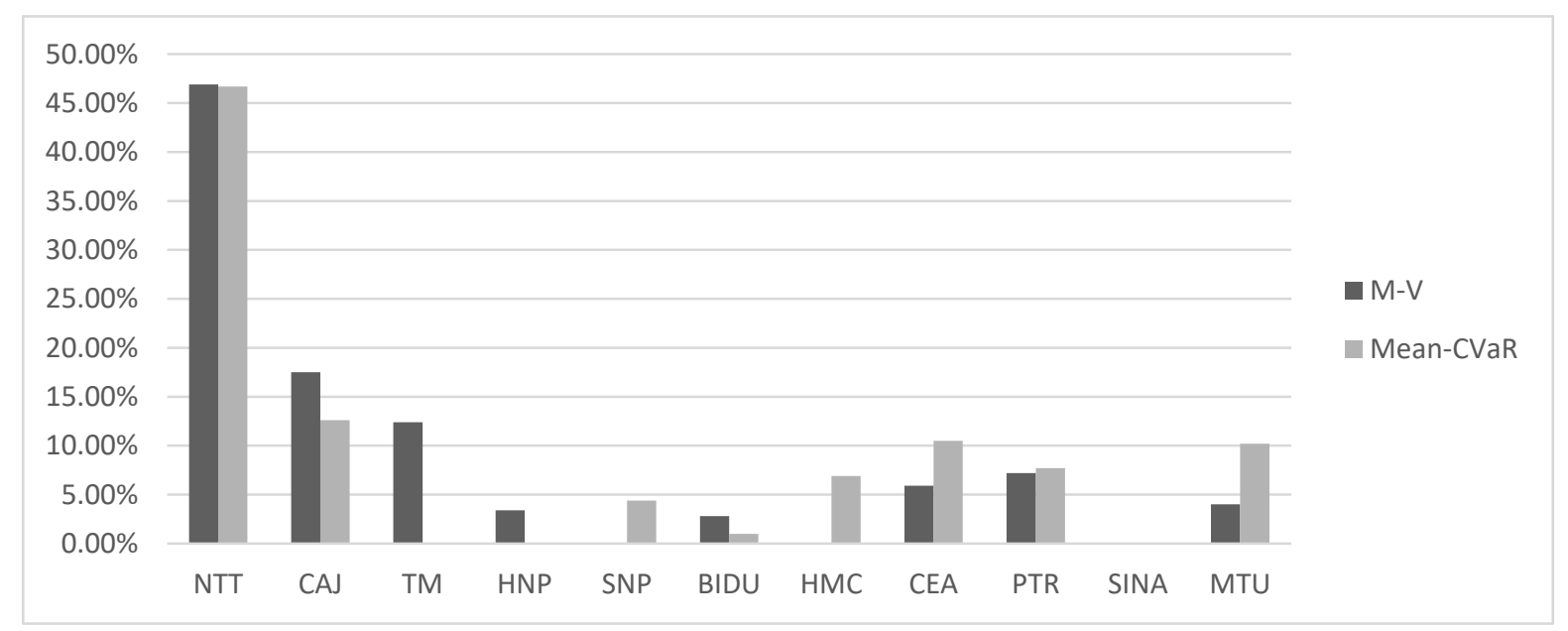

Figure 4.3.2. Asset Allocation Skewed T with 2 Degrees of Freedom

Comparing the two frameworks, one notable difference is the composition of optimal asset. That is, CAJ supersedes TM as the second largest holding in 4.3.2, while both HMC and MTU hold a more important amount of share in the mean-CVaR. Figure 4.3.3 shows the case with 3 degrees of freedom. 


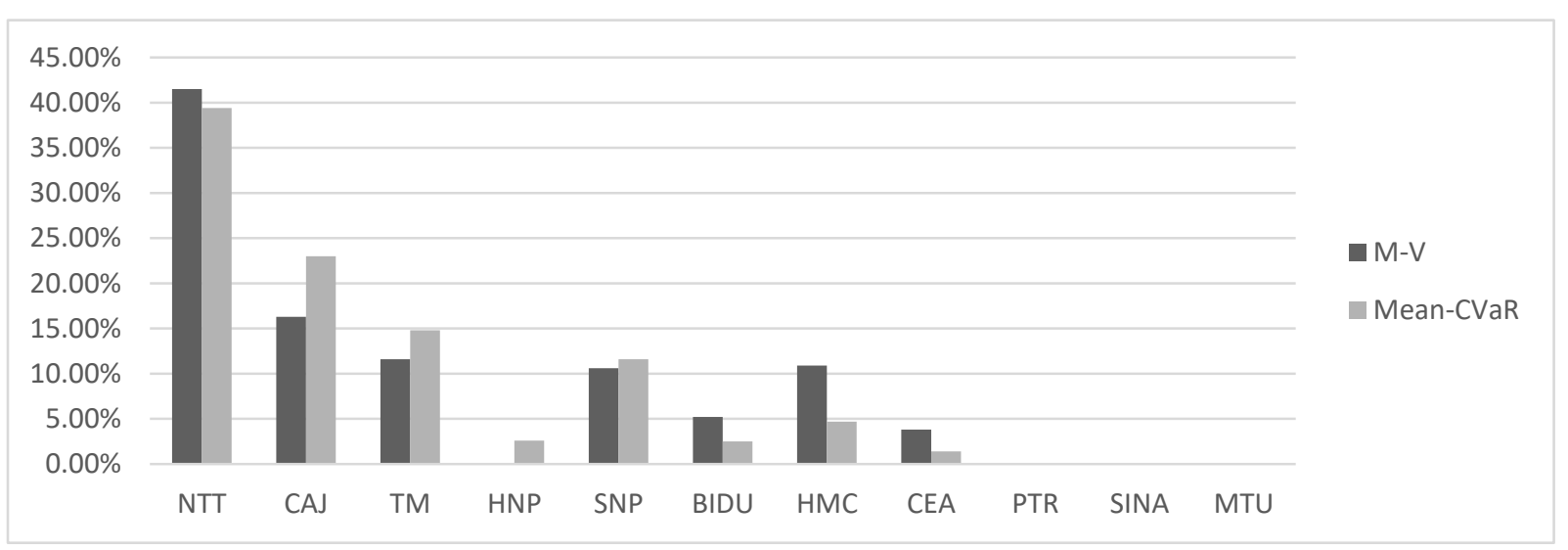

Figure 4.3.3. Asset Allocation Skewed T with 3 Degrees of Freedom

Interestingly, both optimization frameworks yield a more diversified optimal allocation using mean-variance analysis as opposed to mean-CVaR, though the statistical significance of the diversification remains uncertain.

In summary, Table 3 compares the relative performance of each distribution in portfolio selection. To compare the performance of each risk measure, we apply volatility and CVaR within each framework.

Table 3. Realized Volatility and CVaR under Different Distribution Functions

REALIZED VOLATILITY

\begin{tabular}{llllll}
\hline & Historical & Generalized & Optimal & Skewed T & Skewed T \\
& Approach & Hyperbolic & Skewed T & with 2 D.F. & with 3 D.F. \\
M-V & 1.006 & 0.535 & 1.044 & 1.939 & 1.329 \\
MEAN-CVAR & 1.022 & $0.559 \quad$ & 1.051 & 1.987 & 1.342 \\
& & \multicolumn{2}{c}{ REALIZED CVAR } \\
& Historical & Generalized & Optimal & Skewed T & Skewed T \\
& Approach & Hyperbolic & Skewed T & with 2 D.F. & with 3 D.F. \\
M-V & 2.314 & 1.208 & 2.281 & 4.782 & 3.332 \\
MEAN-CVAR & 2.295 & 1.173 & 2.268 & 4.681 & 3.297 \\
\hline
\end{tabular}

The table demonstrates that the hyperbolic distribution $(\mathrm{GH})$ provides the smallest risk in the mean-variance approach, and the same can be found in mean-CVaR framework. On the other hand, the relative performance between mean-CVaR and mean-variance in modeling the portfolio risk, to some extent, depends more on the return distributions with the exception of GH distribution, as evidenced by a mixed risk measure.

\section{Conclusion}

The paper addresses the existence of skewness and fat tail in portfolio selection and investigates its potential effect using a Monte-Carlo simulation technique that applies both mean-variance and mean-CVaR risk optimization frameworks. The empirical results can be summed up as follows: First, the advantage of CVaR relative to variance is not definite, instead the distribution itself could sometimes hold more influence on the outcome. Our result differs from other studies that show mean-CVaR is unconditionally superior to mean-variance framework such as Hofsa (2015). Second, the GH (Generalized Hyperbolic) distribution is shown to outperform the rest in achieving the minimal risk in both frameworks with a diversified optimal allocation. Third, the optimal skewed-t distribution appears to produce an outcome similar to that of the historical method. In conclusion, when dealing with non-normality in financial assets, it is recommended to use the GH (Generalized Hyperbolic) in implementing the portfolio selection in Asian markets, which yields the lowest risk for a given level of target return. The similar findings can be found in Surya and Kurniawan (2014) paper that shows GH distribution provides an excellent fit for the financial return, and it can be extended to the normal and Student's t distributions.

\section{References}

Acerbi, C., \& Tasche, D. (2002). Expected shortfall: a natural coherent alternative to value at risk. Economic notes, 31(2), 379-388. https://doi.org/10.1111/1468-0300.00091

Alexander, G. J., \& Baptista, A. M. (2004). A comparison of VaR and CVaR constraints on portfolio selection with the mean-variance model. Management science, 50(9), 1261-1273. https://doi.org/10.1287/mnsc.1040.0201

Barndorff, N. O. (1977). Exponentially decreasing distributions for the logarithm of particle size. Proc. R. Soc. Lond. A, 353(1674), 401-419. https://doi.org/10.1098/rspa.1977.0041 
Birge, J. R., \& Chavez, B. L. (2016). Portfolio optimization under a generalized hyperbolic skewed t distribution and exponential utility. Quantitative Finance, 16(7), 1019-1036. https://doi.org/10.1080/14697688.2015.1113307

Ho, L. C., Cadle, J., \& Theobald, M. (2008). Portfolio selection in an expected shortfall framework during the recent 'credit crunch'period. Journal of Asset Management, 9(2), 121-137. https://doi.org/10.1057/jam.2008.15

Hofsa, H. (2015). CVaR in Portfolio Optimization: An Essay on the French Market. International Journal of Financial Research, 6. https://doi.org/10.5430/ijfr.v6n2p101

Markowitz, H. (1952). Portfolio Selection. The Journal of Finance, 7(77). https://doi.org/10.2307/2975974

Rockafellar, R. T., \& Uryasev, S. (2000). Optimization of conditional value-at-risk. Journal of risk, 2 , 21-42. https://doi.org/10.21314/JOR.2000.038

Skoglund, J., \& Chen, R. W. (2009) contributions, information and reverse stress testing. The Journal of Risk Model Validation, 3(2), 61-77. https://doi.org/10.21314/JRMV.2009.041

Surya, B. A., \& Kurniawan, R. (2014). Optimal Portfolio Selection Based on Expected Shortfall Under Generalized Hyperbolic Distribution. Asia-Pacific Financial Markets, 21, 193-236. https://doi.org/10.1007/s10690-014-9183-X

\section{Copyrights}

Copyright for this article is retained by the author(s), with first publication rights granted to the journal.

This is an open-access article distributed under the terms and conditions of the Creative Commons Attribution license which permits unrestricted use, distribution, and reproduction in any medium, provided the original work is properly cited. 